\title{
Intervención grupal de promoción de hábitos saludables en el ámbito laboral: Programa IPHASAL
}

\section{Group intervention to promote healthy habits in the workplace: IPHASAL Program}

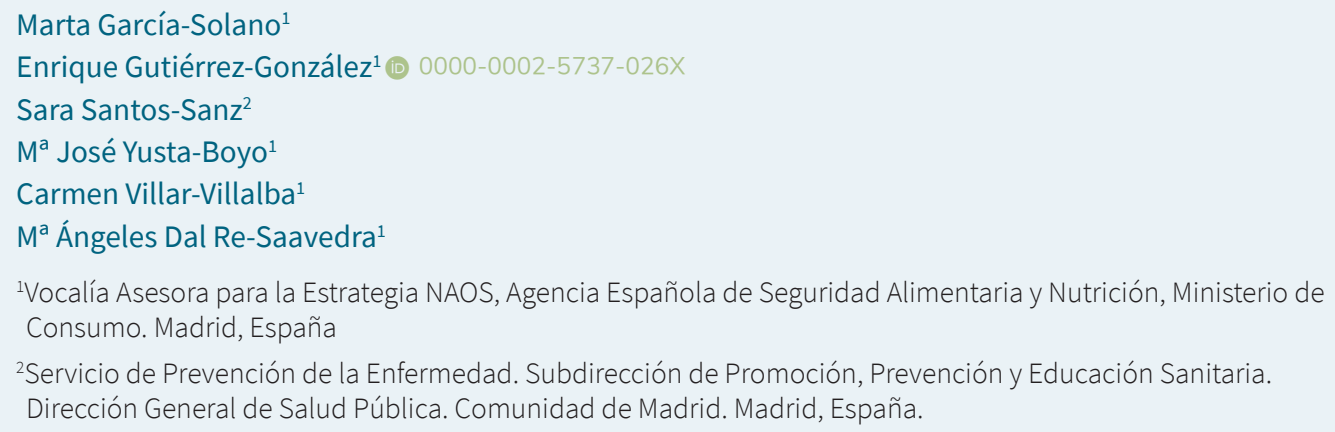

\section{Correspondencia}

Enrique Gutiérrez-González

egutierrez@aesan.gob.es

Recibido: 09.03.2021

Aceptado: 29.03.2021

Publicado: 31.03 .2021

\section{Contribuciones de autoría}

En la concepción y diseño del trabajo y recogida de datos han participado todos los autores. En el análisis e interpretación de los datos han participado Marta García-Solano y Enrique Gutiérrez-González. Todos los autores han participado en la redacción del manuscrito, así como en la revisión crítica del mismo con importantes aportaciones, habiendo aprobado todos ellos la versión final.

\section{Financiación}

El programa IPHASAL fue realizado con los recursos y medios propios de la AESAN, la Fundación Alimentum y las empresas participantes.

\section{Conflicto de intereses}

Los autores declaran no tener ningún conflicto de interés.

\section{How to cite this paper}

García-Solano M, Gutiérrez-González E, Santos-Sanz S, Yusta-Boyo MJ, Villar-Villalba C, Dal Re-Saavedra MA. Intervención grupal de promoción de hábitos saludables en el ámbito laboral: Programa IPHASAL. Med Segur Trab (Internet). 2021;67(262):24-36. doi: 10.4321/s0465-546×2021000100003 


\title{
Resumen
}

Introducción: Las enfermedades cardiovasculares constituyen un grave problema de salud pública. El ámbito laboral es óptimo para intervenir sobre los factores de riesgo cardiovascular. El objetivo es evaluar una intervención grupal de promoción de hábitos saludables (programa IPHASAL) mediante apoyo entre iguales en el ámbito laboral.

Método: En el programa participaron 325 trabajadores de 13 empresas con factores de riesgo cardiovascular (hipertensión, tabaquismo, exceso de peso, inactividad física). Antes y después del programa se midió peso y altura, tensión arterial y se pasó un cuestionario sobre hábitos de salud. El programa consistía en asistir a talleres formativos y sesiones grupales en las que los participantes compartían experiencias, conocimientos y progresos sobre hábitos saludables. El resultado se midió con el Índice Fuster BEWAT (IFB), calculado con la puntuación de 5 variables: tensión arterial, ejercicio, peso, alimentación y tabaco.

Resultados: Los participantes sin criterios de exclusión en el proyecto fueron 273 . El $79 \%$ asistió a 3 o más talleres y el 73,9\% acudió a 3 o más sesiones. Todos los parámetros del IFB mejoraron significativamente tras el programa, siendo el mayor incremento en la actividad física y el menor en el tabaquismo. El IFB total aumentó un 17,4\% (1,5 puntos), siendo superior el aumento en mujeres (19,6\%) que en hombres (14,0\%) y en el grupo más joven (21,6\%).

Conclusiones: La intervención grupal IPHASAL es aplicable al ámbito laboral y contribuye a mejorar el perfil de riesgo cardiovascular, ayudando a los trabajadores a potenciar el cambio hacia estilos de vida saludables.

Palabras clave: riesgo cardiovascular; hábitos saludables; salud laboral; promoción de la salud; apoyo entre iguales.

\begin{abstract}
Introduction: Cardiovascular disease is a major public health problem. The workplace is an optimal setting to intervene on cardiovascular risk factors. The aim is to evaluate a group intervention to promote healthy habits (IPHASAL program) through peer support in the workplace.
\end{abstract}

Method: The program involved 325 workers in 13 companies with cardiovascular risk factors (hypertension, smoking, overweight, physical inactivity). Before and after the program, weight and height, blood pressure were measured and a questionnaire on health habits was administered. The program consisted of training workshops and group sessions in which participants shared experiences, knowledge and progress on healthy habits. The outcome was measured with the Fuster BEWAT Index (FBI), calculated with the score of 5 variables under study: blood pressure, exercise, weight, diet and smoking.

Results: Participants without exclusion criteria in the project were $273.79 \%$ attended 3 or more workshops and $73.9 \%$ attended 3 or more sessions. All FBI parameters improved significantly after the program, with the largest increase in physical activity and the smallest increase in smoking. Total FBI increased by $17.4 \%$ ( 1.5 points), with a higher increase in women (19.6\%) than in men (14.0\%) and in the youngest group (21.6\%).

Conclusions: The IPHASAL group program is applicable to the workplace and contributes to improving the cardiovascular risk profile, helping workers to promote change towards healthy lifestyles.

Keywords: cardiovascular risk; healthy lifestyles; occupational health; health promotion; peer support. 


\section{Introducción}

Las enfermedades no transmisibles (ENT) son responsables de más de dos tercios de todas las muertes que se producen en el mundo, siendo las enfermedades cardiovasculares la principal causa de muerte por ENT en España y en todo el mundo ${ }^{(1,2)}$ por lo que es necesario establecer de forma urgente estrategias que ayuden a reducir este problema de salud pública ${ }^{(3)}$. El exceso de peso, los hábitos alimentarios no saludables, el consumo de tabaco, la inactividad física y la tensión arterial elevada son algunos de sus principales factores de riesgo ${ }^{(4)}$. Según datos de la Encuesta Nacional de Salud de $2017^{(5)}$, en España la prevalencia estimada de sobrepeso y obesidad en adultos era del $37,1 \%$ y $17,4 \%$ respectivamente, el 22,1 \% de la población mayor de 15 años era fumadora, el 19,8 \% tenía hipertensión arterial y el 35,3 $\%$ de la población no alcanzaba el nivel de actividad física saludable recomendado por la OMS.

Las intervenciones dirigidas a la promoción de la salud cardiovascular (práctica regular de actividad física, una alimentación saludable, no fumar y mantener cifras normales de peso y tensión arterial) podrían reducir hasta en un $81 \%$ el riesgo relativo de mortalidad por cualquier causa ${ }^{(6)}$. Estas intervenciones, dirigidas a actuar sobre factores modificables para evitar el desarrollo de ENT o reducir su impacto, se encuentran dentro de los objetivos del Plan de acción Mundial para la Prevención y Control de las ENT 2013-2020 de la OMS ${ }^{(7)}$ y son más eficaces cuando tienen un enfoque poblacional ${ }^{(8)}$.

El apoyo entre iguales («peer support») en el ámbito de la salud se conoce como el apoyo entre personas que tienen las mismas enfermedades o situación de salud y que, por tanto, experimentan situaciones similares ${ }^{(9)}$. Las iniciativas basadas en el apoyo entre iguales ofrecen motivación, aprendizaje y empoderamiento para lograr y mantener comportamientos saludables ${ }^{(10)}$. El apoyo entre iguales ha sido ampliamente utilizado, y se ha demostrado que tiene un impacto positivo en personas que padecen una misma enfermedad, aunque son escasos los ejemplos de intervenciones grupales de apoyo entre iguales para el abordaje de cambios de estilo de vida en personas sanas ${ }^{(11)}$. El ámbito laboral resulta, además, óptimo para el desarrollo de estrategias de promoción de la salud, incidiendo de forma favorable en la salud de los trabajadores, pero repercutiendo positivamente también en las familias, empresas, comunidades y sociedad en general ${ }^{(12)}$.

En 2013 la Agencia Española de Seguridad Alimentaria y Nutrición (AESAN) y la Fundación SHE (Foundation for Science, Health and Education) cofinanciaron y desarrollaron el Programa Fifty-Fifty de intervención comunitaria controlada y aleatorizada basado en una estrategia grupal de apoyo entre iguales o pares, que evidenció la efectividad de este tipo de estrategias para favorecer el cambio de hábitos relacionados con la salud cardiovascular, reduciendo de forma global los factores de riesgo cardiovascular, y de forma significativa la cesación del hábito tabáquico ${ }^{(13)}$. Posteriormente se acreditó como «Modelo de buenas prácticas de intervención de salud comunitaria en la población española adulta para la promoción de hábitos cardiosaludables ${ }^{(14)}$. El programa Fifty-Fifty, además fue también aplicado en el ámbito laboral de forma satisfactoria en dos empresas (Portaventura, 2015 y AMPO, 2016) ${ }^{(15)}$. A pesar de la efectividad de las intervenciones de la promoción de la salud en el ámbito laboral $^{(16)}$, estas no se aplican de forma sistemática en nuestro país y son escasos los ejemplos de su implementación ${ }^{(17)}$.

El objetivo de este trabajo es la evaluación de la implantación de un programa de intervención grupal de promoción de hábitos saludables mediante apoyo entre pares en el ámbito laboral, valorando la efectividad del mismo a la hora de modificar factores de riesgo cardiovascular.

\section{Métodos}

En el programa IPHASAL (Figura 1) participaron 13 empresas de la industria alimentaria pertenecientes a la Fundación Alimentum (FA). La FA proporcionó a los equipos directivos de las empresas participantes información sobre el proyecto y solicitó la designación de un coordinador en cada una, cuya función era facilitar la gestión y desarrollo del programa, bajo la coordinación y supervisión de la AESAN y la FA. Una vez la empresa aceptaba participar, se reclutaban participantes entre los trabajadores a través de los servicios de prevención de riesgos laborales y/o recursos humanos de las diferentes empresas. 
Para este fin las empresas recurrieron a diferentes métodos como un correo electrónico, charlas informativas, intranet, cartelería, etc. La intervención tuvo lugar entre octubre de 2017 y febrero de 2019.

Los criterios de inclusión en el proyecto eran: tener entre 18-65 años y presentar uno o más factores de riesgo: tener sobrepeso u obesidad (IMC $\geq 25 \mathrm{~kg} / \mathrm{m}^{2}$ ), bajo nivel de actividad física (practicar $<150$ minutos de actividad física moderada-intensa a la semana), ser fumador o tener elevada la tensión arterial (Tensión arterial (TA) $\geq 140 / 90 \mathrm{~mm} \mathrm{Hg}$ o recibir tratamiento). Como criterios de exclusión figuraban el embarazo o tener alguna patología crónica diagnosticada (enfermedad cardiovascular o cerebrovascular, diabetes mellitus, patología oncológica, enfermedad inflamatoria intestinal, cualquier otra enfermedad de carácter crónico que requiere tratamiento continúo con fármacos inmunosupresores o tratamiento endocrino-metabólico crónico).

\section{INTERVENCIÓN DE PROMOCIÓN DE HÁBITOS SALUDABLES EN EL ÁMBITO LABORAL}

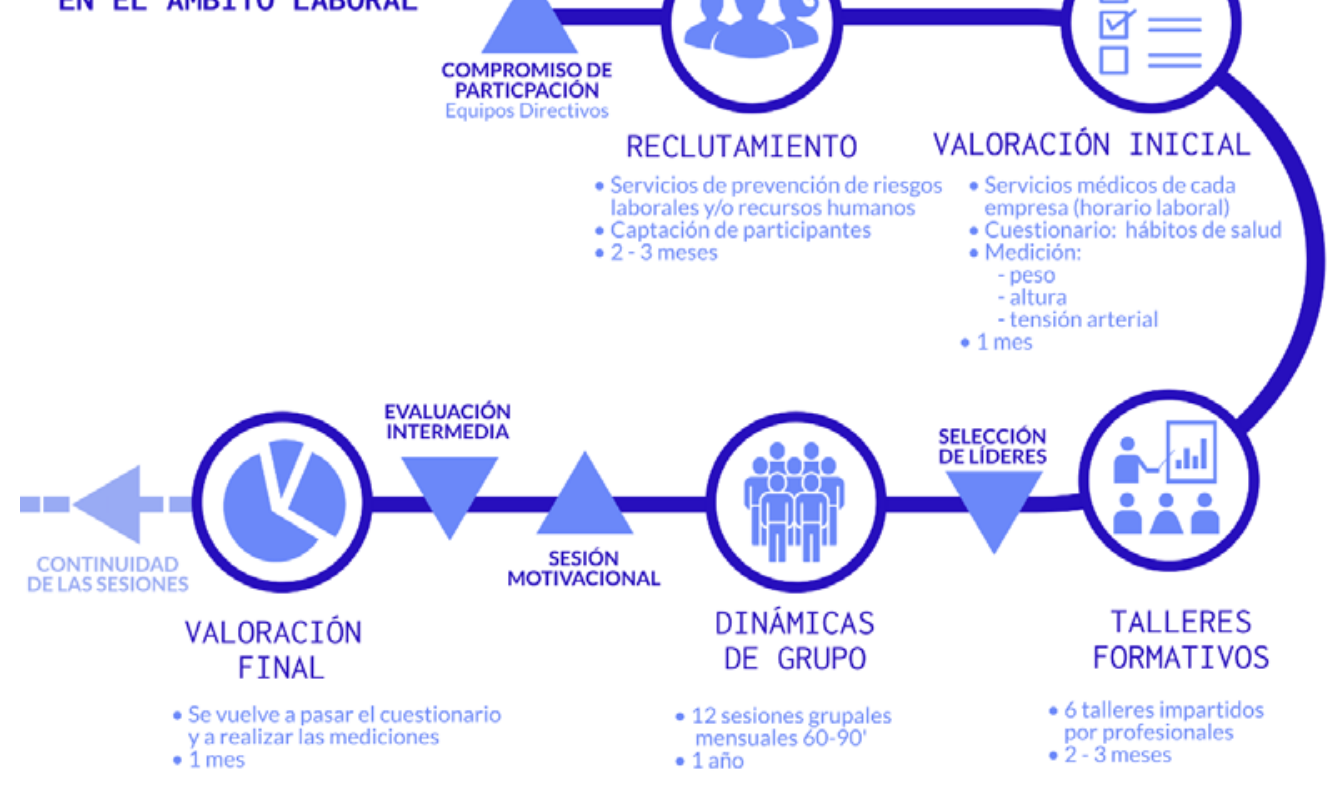

Figura 1: Detalle de las fases del programa IPHASAL.

Una vez habían aceptado participar, previo consentimiento informado, los trabajadores participantes de cada empresa asistían a talleres formativos, 6 en total, impartidos por profesionales propios de la empresa o externos, durante el horario laboral, cuyas temáticas fueron: motivación para el cambio, gestión del estrés, cesación tabáquica, alimentación saludable, actividad física y tensión arterial. A los participantes se les tomaban medidas antropométricas (peso y altura), la tensión arterial y se les pasaba un cuestionario sobre hábitos de salud ${ }^{(18)}$. Estas valoraciones eran llevadas a cabo por los servicios médicos de cada empresa en horario laboral.

Posteriormente, en cada empresa se desarrollaban mensualmente sesiones grupales de los participantes de 60-90 minutos de duración (12 planificadas inicialmente), en horario laboral, en las que explicaban sus experiencias, conocimientos sobre hábitos saludables, sus progresos y las dificultades encontradas. Los líderes de grupo, conocidos como «facilitadores», que a su vez habían sido seleccionados entre los participantes y formados para este fin, dinamizaban estas sesiones y, junto con el resto de participantes, daban apoyo y estimulaban a los demás trabajadores. Cada grupo contaba también 
con un sublíder, que apoyaba al líder y desarrollaba sus funciones cuando estaba ausente. Entre las actividades previstas en las dinámicas del grupo se incluían discusiones en grupo, juegos de roles, lluvia de ideas, técnicas de relajación, diseño de menús o actividades deportivas. En cada reunión, los participantes trataron la gestión emocional, la resolución de problemas, la prevención de recaídas, el control de la dieta y la realización de actividad física, proponiendo a través de estas reflexiones metas alcanzables para mejorar su estilo de vida.

Opcionalmente se podía hacer una sesión motivacional adicional y una evaluación intermedia. Una vez finalizada la intervención, se volvieron a tomar medidas antropométricas y cifras de tensión arterial a los participantes y se volvió a pasar el cuestionario de hábitos de salud.

El resultado principal de la intervención se midió a través del cambio medio, desde la valoración basal hasta la evaluación al final, en un índice calculado a partir de la suma de la puntuación en los valores de las 5 variables principales en estudio: tensión arterial, ejercicio, peso, alimentación y tabaco (Índice Fuster BEWAT, IFB) ${ }^{(13)}$. La puntuación en cada parámetro varía de 0 a 3 (siendo 3 el valor óptimo), oscilando la puntuación global del índice entre 0 y 15 puntos: una puntuación de 0 significa unos hábitos menos saludables y mayor riesgo cardiovascular, mientras que una puntuación de 15 indica unos hábitos más saludables y menor riesgo cardiovascular.

Dado que la efectividad del programa dependía de la adherencia al mismo, se consideró una asistencia mínima de 3 sesiones para evaluar los efectos de la intervención. La adherencia se clasificó como baja para aquellos que realizaron entre 3 y 5 sesiones, media entre 6 y 8 sesiones y alta para los que asistieron a 9 o más sesiones.

En el análisis descriptivo se calcularon medias y desviaciones estándar para las variables cuantitativas, así como recuentos y proporciones para las cualitativas. Se utilizó la prueba de la Chi cuadrado para verificar la asociación entre variables cualitativas y la t de Student para cuantitativas o, si la distribución no era normal, el test de Mann-Whitney. En el caso de comparación de resultados de la intervención con la situación de partida, se utilizaron los tests mencionados previamente para datos apareados. También se evaluaron los factores predictores de mejora del IFB tras la intervención, mediante una regresión logística multivariante. Se consideraron diferencias significativas cuando el valor de $p<0,05$. Los datos fueron proporcionados por la FA de forma anonimizada y analizados mediante el paquete estadístico Stata v.16.

\section{Resultados}

Inicialmente se reclutaron 325 personas de 13 empresas, aunque los empleados de una de las empresas $(n=37)$ fueron excluidos del análisis por no disponerse de los datos individualizados y en 15 de los participantes se detectó algún criterio de exclusión. Los participantes de otras dos empresas $(n=45)$ se excluyeron del análisis ya que no facilitaron datos de sus empleados. El número final de participantes sin criterios de exclusión fue de 273 (84,0\% de la muestra inicial), pertenecientes a 10 empresas. Al finalizar IPHASAL se disponía de información de seguimiento de 186 participantes de 9 empresas que habían asistido a 3 o más sesiones (57,2\%). (Figura 2).

\section{Datos basales}

Las características iniciales de los participantes se muestran en la Tabla 1. La edad media de los participantes era de 45,8 años (mediana 46), con un rango que iba desde los 24 a los 65 años, siendo las mujeres (55,7 \%) significativamente más jóvenes (2,7 años) que los hombres. En relación al rol durante la intervención, la mayoría $(89,6 \%)$ tenía un rol participante durante todo el desarrollo de la misma mientras que un $7,3 \%$ y $3,1 \%$ tenían rol de líder o sublíder respectivamente. 


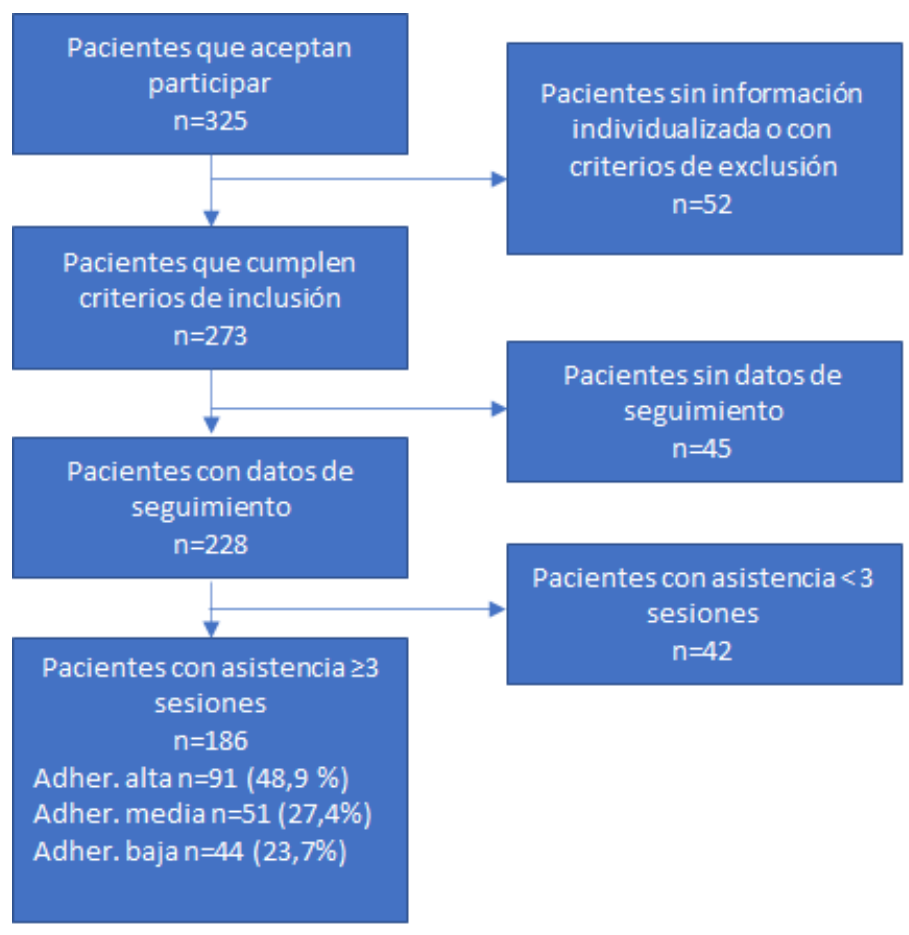

Figura 2: Participantes en el programa IPHASAL.

El 26,5\% de los participantes tenía cifras de TA diastólica (TAD) o TA sistólica (TAS) elevada, presentando las mujeres cifras significativamente inferiores tanto de TAD como TAS a las de los hombres, aumentando también las cifras de TAD y TAS significativamente con la edad. El 29,3\% de los participantes declaraban realizar menos de 10 minutos semanales de actividad física moderada-intensa, frente al $27,5 \%$ que declaraba dedicar más de 150 minutos, sin diferencias significativas por sexo ni edad. El $31,2 \%$ de los participantes tenían normopeso, el 34,6\% sobrepeso y el 31,2 \% obesidad, siendo significativamente superior el IMC de hombres respecto a mujeres, aumentando también con la edad. Un 43,6 \% de los participantes declaraba tomar entre 1 y 2 piezas/raciones de fruta y verdura al día y otro $40,7 \%$ consumía entre 3 y 4 , mientras que un $7,7 \%$ de los participantes afirmaba no tomar ningún tipo de fruta y verdura a diario, sin diferencias por sexo, pero sí por edad, observando el menor consumo en el grupo más joven. Aproximadamente dos tercios $(65,9 \%)$ de los participantes nunca habían fumado, el 22,7 \% eran fumadores antes de la intervención y un 11,4 \% eran ex-fumadores, sin diferencias por sexo, pero sí por edad, siendo significativamente menor el porcentaje de fumadores en el grupo de mayor edad. La media de la puntuación del IFB del total de participantes era de 8,7, siendo significativamente superiores las puntuaciones de las mujeres respecto a los hombres y disminuyendo también de forma significativa con la edad.

\section{Desarrollo de la intervención}

El 79 \% de los participantes asistió a 3 o más talleres y un 39,2 \% completó los 6 talleres, siendo 4,3 talleres la asistencia media por participantes, sin diferencias por sexo, aunque sí por edad, aumentando la asistencia a talleres con la edad.

El 73,9 \% de los participantes sin criterios de exclusión asistió a 3 o más sesiones mensuales, que era el mínimo considerado para evaluar la intervención, por lo que el 27,1\% de los participantes fue excluido de la evaluación post-intervención por su muy baja asistencia (<3 sesiones) (Figura 2). El 34,3\% de los 
participantes sin criterios de exclusión tuvieron una adherencia alta al proyecto ya que asistieron a 9 o más sesiones, mientras que el $20,2 \%$ y el $18,3 \%$ tuvieron una asistencia media (6-8 sesiones) y baja (3-5 sesiones), respectivamente, con una participación media de 6,7 sesiones por participante.

Tabla 1: Características de los participantes antes y después de la intervención.

\begin{tabular}{|c|c|c|}
\hline & Pre-intervención $(n=273)$ & Post-intervención ( $n=186$ ) \\
\hline \multicolumn{3}{|l|}{ Edad } \\
\hline Años (media, DE) & $45,8(9,2)$ & $45,8(8,7)$ \\
\hline \multicolumn{3}{|l|}{ Grupos de edad } \\
\hline 24-35 años (n, \%) & $38(15,0 \%)$ & $23(12,9 \%)$ \\
\hline 36-45 años (n, \%) & $88(34,8 \%)$ & $63(35,4 \%)$ \\
\hline $46-55$ años $(n, \%)$ & $80(31,6 \%)$ & $59(33,2 \%)$ \\
\hline $56-65$ años (n, \%) & $47(18,6 \%)$ & $33(18,5 \%)$ \\
\hline \multicolumn{3}{|l|}{ Sexo } \\
\hline Mujeres (n, \%) & $152(55,7 \%)$ & $108(58,1 \%)$ \\
\hline \multicolumn{3}{|l|}{ Tensión arterial (n, \%) } \\
\hline TAD y TAS normales (<80 y <120 mmHg) & $124(45,6 \%)$ & $72(43,1 \%)$ \\
\hline TAD o TAS elevada ( $\geq 90 \circ \geq 140 \mathrm{mmHg}$ ) & $72(26,4 \%)$ & $33(19,8 \%)$ \\
\hline \multicolumn{3}{|l|}{ Actividad física moderada-intensa } \\
\hline Minutos/semana (media, DE) & $119,1(123,4)$ & $152,0(133,4)$ \\
\hline$<10$ minutos/semana $(n, \%)$ & $80(29,3 \%)$ & $40(21,6 \%)$ \\
\hline$>150$ minutos/semana $(n, \%)$ & $75(27,5 \%)$ & $82(44,3 \%)$ \\
\hline \multicolumn{3}{|l|}{ Situación ponderal } \\
\hline IMC, $\mathrm{kg} / \mathrm{m}^{2}$ (media, DE) & $28,1(5,5)$ & $28,4(5,3)$ \\
\hline Normopeso (n, \%) & $84(31,2 \%)$ & $44(26,4 \%)$ \\
\hline Sobrepeso (n, \%) & $93(34,6 \%)$ & $69(41,3 \%)$ \\
\hline Obesidad (n, \%) & $92(34,2 \%)$ & $54(32,3 \%)$ \\
\hline \multicolumn{3}{|l|}{ Consumo frutas y verduras } \\
\hline Piezas / día (media, DE) & $2,4(1,4)$ & $3,1(1,6)$ \\
\hline No consumen (n, \%) & $21(7,7 \%)$ & $21(11,3 \%)$ \\
\hline 1-2 piezas /día (n, \%) & $119(43,6 \%)$ & $30(16,1 \%)$ \\
\hline 3-4 piezas/día (n, \%) & $111(40,7 \%)$ & $103(55,4 \%)$ \\
\hline$\geq 5$ piezas/día $(n, \%)$ & $22(8,0 \%)$ & $32(17,2 \%)$ \\
\hline \multicolumn{3}{|l|}{ Tabaco } \\
\hline No fumador (n, \%) & $180(65,9 \%)$ & $129(69,4 \%)$ \\
\hline Exfumador (n, \%) & $31(11,4 \%)$ & $30(16,1 \%)$ \\
\hline Fumador (n, \%) & $62(22,7 \%)$ & $27(14,5 \%)$ \\
\hline Cigarrillos/día fumadores (media, DE) & $10,3(7,1)$ & $6,3(5,7)$ \\
\hline
\end{tabular}




\section{Datos post-intervención}

Tras la intervención, se disponía de información individualizada de 228 participantes de 10 empresas (79,2 \% de la muestra inicial), aunque de ellos 42 fueron excluidos del análisis debido a la muy baja adherencia (asistencia a $<3$ sesiones), por lo que se evaluaron los datos tras la intervención de 186 participantes de 9 empresas (57,2 \% muestra inicial) (Figura 2).

Las características de los participantes después de la intervención se muestran en la Tabla 1, siendo la distribución por edad y sexo muy similar a la previa a la intervención. En comparación con la situación basal, había un menor porcentaje de hipertensos (19,8\%) y de participantes que declaraban realizar menos de 10 minutos semanales de actividad física moderada-intensa $(21,6 \%)$ y un mayor porcentaje de participantes con sobrepeso $(41,3 \%)$ y de trabajadores que consumía 3 o más piezas de fruta y verdura al día (72,6\%), aunque también aumentaba el porcentaje de los que afirmaban no tomar ningún tipo de fruta y verdura a diario $(11,3 \%)$. El porcentaje de fumadores $(14,5 \%)$ era inferior y el de exfumadores superior $(16,1 \%)$.

Tabla 2: Puntuación (media y desviación estándar) en parámetros del índice Fuster-BEWAT (IFB) en el total de la muestra, por sexo y edad.

\begin{tabular}{|c|c|c|c|c|}
\hline Total & $\begin{array}{l}\text { Pre-intervención } \\
\qquad(\mathrm{n}=186)\end{array}$ & $\begin{array}{l}\text { Post-intervención } \\
\qquad(\mathrm{n}=186)\end{array}$ & Diferencia & $\mathbf{p}$ \\
\hline $\mathrm{B}^{*}$ & $1,78(1,29)$ & $1,96(1,22)$ & $0,18(1,02)$ & $<0,05$ \\
\hline$E^{\star}$ & $1,56(1,16)$ & $1,93(1,18)$ & $0,37(1,27)$ & $<0,05$ \\
\hline$W^{*}$ & $1,06(1,16)$ & $1,34(1,14)$ & $0,28(0,68)$ & $<0,05$ \\
\hline$A^{*}$ & $1,53(0,72)$ & $1,79(0,86)$ & $0,26(0,93)$ & $<0,05$ \\
\hline$T^{*}$ & $2,60(0,81)$ & $2,73(0,66)$ & $0,13(0,46)$ & $<0,05$ \\
\hline Total IFB & $8,63(2,62)$ & $10,13(2,54)$ & $1,50(2,00)$ & $<0,05$ \\
\hline \multicolumn{5}{|l|}{ IFB por Sexo } \\
\hline Hombres & $8,15(2,71)$ & $9,29(2,58)$ & $1,14(1,98)$ & $<0,05$ \\
\hline Mujeres & $8,96(2,52)$ & $10,72(2,3)$ & $1,76(1,98)$ & $<0,05$ \\
\hline \multicolumn{5}{|c|}{ IFB por grupo de edad } \\
\hline 24-35 años & $9,28(2,47)$ & $11,28(1,90)$ & $2,00(1,57)$ & $<0,05$ \\
\hline 36-45 años & $9,15(2,91)$ & $10,42(2,72)$ & $1,27(1,92)$ & $<0,05$ \\
\hline 46-55 años & $7,96(2,66)$ & $9,65(2,63)$ & $1,69(2,06)$ & $<0,05$ \\
\hline 56-65 años & $8,21(1,85)$ & $9,75(1,99)$ & $1,54(2,25)$ & $<0,05$ \\
\hline \multicolumn{5}{|c|}{ IFB por adherencia } \\
\hline Baja & $8,68(2,18)$ & $9,29(2,49)$ & $0,62(1,97)$ & 0,08 \\
\hline Media & $7,46(2,63)$ & $9,61(2,71)$ & $2,15(2,74)$ & $<0,05$ \\
\hline Alta & $9,17(2,63)$ & $10,72(2,34)$ & $1,55(1,96)$ & $<0,05$ \\
\hline
\end{tabular}

*B (tensión arterial), E (actividad física), W (situación ponderal), A (consumo frutas y verduras), T (tabaco).

Para la comparación de los parámetros del IFB se tuvo solo en cuenta a aquellos participantes con información antes y después de la intervención ( $n=186$ ) (Tabla 2). Todos los parámetros del IFB mejoraron tras la intervención de forma significativa, siendo el mayor incremento, de 0,37, en la puntuación media del parámetro $\mathrm{E}$ (actividad física) y el menor, de 0,13 , en el parámetro T (hábito tabáquico). La puntuación media de la situación ponderal (parámetro W), el consumo de frutas y verduras (parámetro A) y el hábito tabáquico (parámetro T) mejoraron significativamente en ambos sexos y en todos los 
grupos de edad, mientras que la puntuación media del parámetro B (Tensión arterial) y E (actividad física) mejoraron de forma significativa sólo en las mujeres. El IFB total aumentó un 17,4\% de forma significativa (1,50 en valor absoluto) en el total de la muestra, siendo superior el aumento en mujeres $(19,6 \% ; 1,76)$ que en hombres $(14,0 \% ; 1,14)$. Por grupos de edad, se observó un mayor incremento $(21,6 \% ; 2,00)$ en el grupo más joven (24-35 años). En relación a la adherencia, el incremento fue superior en los grupos de adherencia media y alta, no siendo significativa la mejoría en los participantes con baja adherencia.

Como factor predictor de mejoría en el IFB solo resultó significativo el sexo (mujer), no alcanzando significación estadística otras variables como la edad, la adherencia (número de sesiones) o número de talleres.

\section{Discusión}

En este artículo se presentan los resultados de una intervención de promoción de hábitos saludables en el ámbito laboral basada en el programa Fifty-Fifty, observando una mejoría en los hábitos relacionados con la salud cardiovascular en los empleados que participaron en la intervención, de forma similar a lo evidenciado con el programa original desarrollado en población general ${ }^{(13)}$. Este cambio positivo en los hábitos fue cuantificado mediante el cambio en la puntuación del IFB, que ha demostrado ser un predictor de la presencia de aterosclerosis subclínica ${ }^{(19)}$ y que además puede servir como elemento motivador para los participantes en una intervención de este tipo, por ayudar a visibilizar la mejora en los hábitos. En nuestro estudio todas las variables incluidas en el índice mejoraron significativamente, aunque el parámetro que experimentó un mayor incremento fue el ejercicio físico, a diferencia del programa original, donde fue el tabaco, si bien la proporción de fumadores era superior a la de esta intervención ${ }^{(13)}$.

Una revisión sistemática y meta-análisis de ensayos clínicos de intervenciones para modificar factores de riesgo cardiovascular en trabajadores ${ }^{(16)}$, entre las que se incluían modificaciones nutricionales y actividad física, concluyó que las intervenciones eran efectivas para reducir la tensión arterial, tanto sistólica como diastólica, y el IMC. De los estudios incluidos, solo uno estaba basado completamente en una intervención grupal, dirigida únicamente a modificaciones nutricionales ${ }^{(20)}$, a diferencia del presente estudio que intervenía en aspectos variados como la alimentación, la actividad física y el hábito tabáquico. Aunque existen otras intervenciones similares desarrolladas en el ámbito laboral aplicadas de forma individual con reducciones significativas del riesgo cardiovascular ${ }^{(21)}$, las intervenciones grupales, basadas en el apoyo entre pares tienen la ventaja de mejorar de forma efectiva los conocimientos, las actitudes, creencias y percepciones, mejorando además las conexiones sociales y el compromiso, con resultados prometedores en el campo de la promoción de la salud ${ }^{(11)}$.

El ámbito laboral puede proporcionar un entorno muy favorable para desarrollar actuaciones de promoción de la salud en el marco de la estrategia de control de las enfermedades no transmisibles más prevalentes, las cuales afectan también a población trabajadora. La OMS en su $60^{\text {a }}$ Asamblea Mundial de la Salud a través del plan de acción mundial sobre la salud de los trabajadores 2008-2017 instó a los países miembros a fomentar en los trabajadores una dieta sana y actividad física con el fin de proteger y promover la salud en el lugar de trabajo ${ }^{(22)}$.

La Estrategia Española de Seguridad y Salud en el Trabajo 2015-2020 incluye dentro de sus objetivos impulsar la cultura de la salud fomentando hábitos saludables en el entorno laboral ${ }^{(23)}$. En esta Estrategia se analizó una submuestra de trabajadores de la población estudiada en la Encuesta Nacional de Salud de España 2011/12. Entre los resultados de este análisis realizado por el Instituto Nacional de Seguridad e Higiene en el Trabajo (INSHT) en población ocupada entre 16 y 67 años ( $n=8.640$ ) destacaba, en el marco de hábitos y estilos de vida, que el $34,0 \%$ realizaba un trabajo sedentario, un $73,7 \%$ no realizaba ejercicio físico, un $29,4 \%$ fumaba a diario y un $31,4 \%$ presentaba exceso de peso, que representa el perfil de participantes de nuestra intervención, en la que las cifras eran aún superiores, ya que tener algún factor de riesgo era precisamente un criterio de inclusión. 
La Red Europea de Promoción de la Salud en los Lugares de Trabajo (European Network for Workplace Health Promotion, ENWHP), impulsó una iniciativa llamada «MOVE EUROPE (2007-2009)», con el fin de avalar Buenas Prácticas en las intervenciones de promoción de la salud en el trabajo relacionadas con los hábitos saludables ${ }^{(24)}$. Esta iniciativa describía una serie de condiciones que debe cumplir una intervención de promoción de la salud en el ámbito laboral para ser implementada con éxito, entre las que figuran contar con el apoyo y participación de la dirección, la implicación de los trabajadores, el uso óptimo de los recursos disponibles, la facilidad de acceso a las intervenciones, la observación y evaluación de resultados o una comunicación continua y abierta a todos los trabajadores. El proyecto IPHASAL, basado en el programa Fifty-Fifty ${ }^{(13)}$, reúne estas condiciones necesarias para garantizar la calidad de un programa de promoción de la salud en los lugares de trabajo, centrándose en los principales factores de riesgo de las enfermedades crónicas más prevalentes.

La promoción de la salud en el trabajo, incluida en el Real Decreto 843/2011, de 17 de junio ${ }^{(25)}$, por el que se establecen los criterios básicos sobre la organización de recursos para desarrollar la actividad sanitaria de los servicios de prevención, no solo beneficia a los empleados y a sus familias, así como a la comunidad, sino también a la empresa, contribuyendo a incrementar la productividad y disminuir el absentismo laboral(24).

Para optimizar los resultados de las actividades de promoción de la salud en el ámbito laboral es necesario un enfoque integral, no solo centrado en modificar hábitos de salud sino también en facilitar las opciones saludables en el entorno. Según datos del Barómetro Sanitario 2014 del Centro de Investigaciones Sociológicas, el 32,3\% de las personas trabajadoras tenían acceso a iniciativas de promoción de una alimentación saludable en el centro de trabajo mientras que el 17,9\% tenía acceso a iniciativas de promoción de actividad física en el centro de trabajo, siendo ambas cifras ligeramente superiores con respecto al Barómetro Sanitario de $2010^{(26)}$. En la presente intervención se abordaban hábitos saludables sobre alimentación, ejercicio físico y tabaquismo, que se pueden potenciar favoreciendo ofertas concretas en las empresas como menús saludables en los comedores y cafeterías, máquinas de vending con productos de perfil más saludable, programas de actividades deportivas en las empresas o facilitando el uso de instalaciones deportivas en el trabajo o de localización cercana, entre otras.

Para el desarrollo de cualquier intervención de promoción de la salud en el ámbito laboral es fundamental la colaboración de la empresa, siendo imprescindible que la organización apoye las actividades derivadas de la intervención. Igualmente, se deben poner a disposición los recursos necesarios, aprovechando aquellos que sean propios de las empresas para reducir costes, así como la proximidad de la intervención a los puestos de trabajo, permitiendo que los empleados se involucren más en la misma. Como se ha visto en esta intervención, así como en otras previas, la motivación y la adherencia al programa están asociadas a una mayor consecución de los objetivos planteados ${ }^{(13,27)}$. Otro aspecto clave es la designación de un coordinador en la empresa, que puede ser el responsable del servicio de prevención de riesgos laborales, aprovechando la estructura y organización de las actividades de promoción de la salud en las empresas.

Entre las fortalezas de la intervención está su enfoque integral para la mejora de hábitos de salud (alimentación y actividad física) y el autocontrol de los factores de riesgo cardiovascular (exceso de peso, tensión arterial y tabaquismo). Este enfoque es novedoso dada la metodología empleada en la intervención para conseguir un cambio de hábitos ya que, además de una intervención formativa, se basa en el apoyo entre iguales (grupo de pares o grupo entre iguales o peer-group), que puede contribuir a reforzar los resultados y contribuye al mantenimiento de los hábitos saludables. Como limitaciones, la pérdida en el seguimiento o la exclusión por muy baja adherencia de algunos participantes podría afectar la interpretación de los resultados, si bien análisis de sensibilidad realizados (datos no mostrados) muestran resultados similares incluyendo también a los participantes de muy baja adherencia. También es posible que parte del efecto observado sea debido a un sesgo de atención (efecto Hawthorne) o sesgo de deseabilidad social, ya que los individuos al sentirse observados pueden modificar por este motivo sus hábitos, aunque este efecto es más frecuente al inicio de las intervenciones y suele desaparecer a los pocos meses. Otro aspecto limitante es que aún se desconoce la persistencia en el tiempo de la mejoría lograda en los hábitos una vez que cesan las dinámicas de grupo y el posible efecto de actuaciones de refuerzo. 
La intervención grupal IPHASAL, basada en el Programa Fifty-Fifty, es aplicable al ámbito laboral y contribuye a mejorar el perfil de riesgo cardiovascular en el entorno laboral, tratándose de una herramienta que facilita la construcción de ambientes de trabajo saludables, dotando de recursos personales de salud a los trabajadores y ayudándoles a potenciar el cambio hacia estilos de vida saludables. Es necesario contar con el compromiso de la empresa, servicios médicos y los propios trabajadores para garantizar el éxito de la intervención, así como evaluar y difundir sus resultados.

\section{Agradecimientos}

Agradecemos al Dr. Valentín Fuster y su equipo investigador el diseño del programa de intervención comunitaria «Fifty-Fifty», en el que se ha basado esta «Intervención de promoción de hábitos saludables en el ámbito laboral (Programa IPHASAL)».

Agradecemos también a la Fundación Alimentum por su labor de coordinación e impulso de la intervención y a todas las empresas, a los Servicios de Recursos Humanos y de Prevención de Riesgos Laborales por su colaboración y a los trabajadores que han participado en esta intervención.

\section{Bibliografía}

1. Global, regional, and national age-sex specific mortality for 264 causes of death, 1980-2016: a systematic analysis for the Global Burden of Disease Study 2016. Lancet. 2017;390(10100):1151-210. doi: 10.1016/S0140-6736(17)32152-9

2. Instituto Nacional de Estadística. Estadística de defunciones según la causa de muerte [Internet]. 2018 [citado 25 de febrero de 2020]. Disponible en: https://www.ine.es/dyngs/INEbase/es/operacion. htm?c=Estadistica_C\&cid=1254736176780\&menu=ultiDatos\&idp $=1254735573175$

3. Gaye B, Lloyd-Jones DM. Primordial prevention of cardiovascular disease: Several challenges remain. Int J Cardiol. 2019;274:379-80. doi: 10.1016/j.ijcard.2018.09.063

4. Francula-Zaninovic S, Nola IA. Management of Measurable Variable Cardiovascular Disease' Risk Factors. Curr Cardiol Rev. 2018;14(3):153-63. doi: 10.2174/1573403X14666180222102312

5. Ministerio de Sanidad, Consumo y Bienestar Social - Portal Estadístico del SNS - Encuesta Nacional de Salud de España 2017 [Internet]. [citado 25 de febrero de 2020]. Disponible en: https://www.mscbs. gob.es/estadEstudios/estadisticas/encuestaNacional/encuesta2017.htm

6. Ahmed HM, Blaha MJ, Nasir K, Jones SR, Rivera JJ, Agatston A, et al. Low-risk lifestyle, coronary calcium, cardiovascular events, and mortality: results from MESA. Am J Epidemiol. 2013;178(1):12-21. doi: $10.1093 /$ aje/kws453

7. WHO | Global Action Plan for the Prevention and Control of NCDs 2013-2020 [Internet]. WHO. World Health Organization; [citado 11 de mayo de 2020]. Disponible en: http://www.who.int/nmh/events/ ncd_action_plan/en/

8. Karwalajtys T, Kaczorowski J. An integrated approach to preventing cardiovascular disease: community-based approaches, health system initiatives, and public health policy. Risk Manag Healthc Policy. 2010;3:39-48. doi: 10.2147/RMHP.S7528

9. Dennis CL. Peer support within a health care context: a concept analysis. Int J Nurs Stud. 2003;40(3):321-32. doi: 10.1016/s0020-7489(02)00092-5

10. Funnell MM. Peer-based behavioural strategies to improve chronic disease self-management and clinical outcomes: evidence, logistics, evaluation considerations and needs for future research. Fam Pract. 2010;Suppl 1(Suppl 1):i17-22. doi: 10.1093/fampra/cmp027 
11. Ramchand R, Ahluwalia SC, Xenakis L, Apaydin E, Raaen L, Grimm G. A. Systematic review of peer-supported interventions for health promotion and disease prevention. Prev Med. 2017;101:15670. doi: 10.1016/j.ypmed.2017.06.008

12. Calvo-Bonacho E, Catalina-Romero $C$. Health promotion at the workplace. Hipertens Riesgo Vasc. 2015;32(3):97-9. doi: 10.1016/j.hipert.2015.06.002

13. Gómez-Pardo E, Fernández-Alvira JM, Vilanova M, Haro D, Martínez R, Carvajal I, et al. A Comprehensive Lifestyle Peer Group-Based Intervention on Cardiovascular Risk Factors: The Randomized Controlled Fifty-Fifty Program. J Am Coll Cardiol. 2016;67(5):476-85. doi: 10.1016/j.jacc.2015.10.033

14. Agencia Española de Consumo, Seguridad Alimentaria y Nutrición. Estandarización de un modelo de buenas prácticas de intervención de salud comunitaria en la población española adulta para la promoción de hábitos cardiosaludables. Madrid, España: Ministerio de Sanidad, Servicios Sociales e Igualdad; 2015.

15. Programa Fifty Fifty. Participantes [Internet]. [citado 20 de mayo de 2020]. Disponible en: http:// programafiftyfifty.org/index.php?page=recursos\&sec $=6$

16. Hwang WJ, Kang SJ. Interventions to Reduce the Risk of Cardiovascular Disease among Workers: A Systematic Review and Meta-Analysis. Int J Environ Res Public Health. 2020;17(7):2267. doi: 10.3390/ ijerph17072267

17. San Mauro-Martin I, Sanz-Rojo S, Becerra X, Garicano-Vilar E. Successful Implementation of a Mediterranean Weight Loss Program to Prevent Overweight and Obesity in the Workplace. J Occup Environ Med. 2019;61(8):e329-32. doi: 10.1097/JOM.0000000000001628

18. Intervención de promoción de hábitos saludables en el ámbito laboral (IPHASAL). Agencia Española de Seguridad Alimentaria y Nutrición. Ministerio de Consumo. Madrid, 2020. [Internet]. [citado 11 de enero de 2021]. Disponible en: https://www.aesan.gob.es/AECOSAN/docs/documentos/nutricion/ IPHASAL.pdf

19. Fernández-Alvira JM, Fuster V, Pocock S, Sanz J, Fernández-Friera L, Laclaustra M, et al. Predicting Subclinical Atherosclerosis in Low-Risk Individuals: Ideal Cardiovascular Health Score and Fuster-BEWAT Score. J Am Coll Cardiol. 2017;70(20):2463-73. doi: 10.1016/j.jacc.2017.09.032

20. Salinardi TC, Batra P, Roberts SB, Urban LE, Robinson LM, Pittas AG, et al. Lifestyle intervention reduces body weight and improves cardiometabolic risk factors in worksites123. Am J Clin Nutr. 2013;97(4):667-76. doi: 10.3945/ajcn.112.046995

21. Mastrangelo G, Marangi G, Bontadi D, Fadda E, Cegolon L, Bortolotto M, et al. A worksite intervention to reduce the cardiovascular risk: proposal of a study design easy to integrate within Italian organization of occupational health surveillance. BMC Public Health. 2015;15:12. doi: 10.1186/s12889-0151375-4

22. OMS | Salud de los trabajadores: plan de acción mundial 2008-2017 [Internet]. WHO. World Health Organization; [citado 11 de mayo de 2020]. Disponible en: http://www.who.int/phe/publications/workers_health_global_plan/es/

23. Instituto Nacional de Seguridad e Higiene en el Trabajo. Estrategia Española de Salud y Seguridad en el Trabajo 2015-2020. Madrid, España: Ministerio de Empleo y Seguridad Social; 2015.

24. European Network Workplace Health Promotion. Move Europe. A Campaign for the Improvement of Lifestyle-related Workplace Health Promotion in Europe. Business Report. 2009 [Internet]. [citado 12 de mayo de 2020]. Disponible en: https://www.enwhp.org/resources/toolip/doc/2018/09/20/final-move-europe-business-report.pdf

25. Real Decreto 843/2011, de 17 de junio, por el que se establecen los criterios básicos sobre la organización de recursos para desarrollar la actividad sanitaria de los servicios de prevención. [Internet]. Disponible en: https://www.boe.es/buscar/act.php?id=BOE-A-2011-11428 
26. Ministerio de Sanidad, Consumo y Bienestar Social - Portal Estadístico del SNS - Estadisticas y Estudios - Barómetro Sanitario [Internet]. [citado 13 de mayo de 2020]. Disponible en: https://www.mscbs. gob.es/estadEstudios/estadisticas/inforRecopilaciones/barometro/home.htm

27. Fernandez-Jimenez R, Jaslow R, Bansilal S, Diaz-Munoz R, Fatterpekar M, Santana M, et al. Different Lifestyle Interventions in Adults From Underserved Communities: The FAMILIA Trial. J Am Coll Cardiol. 2020;75(1):42-56. doi: 10.1016/j.jacc.2019.10.021 